\title{
Approche méthodologique de la distribution spatiale des algues épiphytiques sur l'appareil foliaire d'Apium nodiflorum (L.) Lag. (Apiaceae)
}

\author{
K. Comte ${ }^{1}$ \\ S. Fayolle ${ }^{1}$ \\ A.Cazaubon ${ }^{1}$ \\ M. Roux ${ }^{2}$
}

Mots-clés : Algues épiphytiques, distribution spatiale, méthodologie, Apium nodiflorum.

Une étude de la distribution spatiale épiphytique sur une plante aquatique commune des sources karstiques méditerranéennes a été effectuée à l'échelle du microhabitat, sur différentes feuilles d'Apium nodiflorum. Des découpages longitudinaux et transversaux sur toute la longueur du foliole ont été réalisés et analysés séparément.

Hormis de fortes variations liées à chaque individu macrophytique, les premiers résultats, obtenus sur 48 folioles, prélevés en automne et en été, révèlent l'existence d'une microrépartition effective et non stochastique des épiphytes sur les différentes parties architecturales de la plante-hôte, ainsi qu'une homogéneité des communautés algales sur la hauteur totale de la plante.

Cette approche méthodologique a permis de mettre en évidence des microzones représentatives de la richesse spécifique et de la biomasse totale du peuplement épiphytique. Cette approche pourrait faire l'objet d'études élargies à d'autres macrophytes et être utilisée par les limnologues comme outil d'observation du peuplement épiphytique en rivières.

Methodological approach of a spatial distribution pattern of epiphytic algae on Apium nodiflorum leaves (L.) Lag. (Apiaceae)

Keywords : Epiphytic algae, spatial distribution, methodological approach, Apium nodiflorum.

A microhabitat scale study of spatial epiphytic distribution of a common Apiaceae of karstic mediterranean springs has been carried out, on the leaf system of Apium nodiflorum. Longitudinal and transverse zones along the leaf have been determined and separatetly analysed.

In spite of a physiologic variability inherent in each sample, initial results, based on a 48 leaflets sample, for each stage, reveal a non-random distribution of epiphytes on different architectural elements of the host-plant. A homogeneity of algal communities is noted on the whole plant height from surface to base. The several scales necessary for this methodological study clearly show existence of microzones which are quantitatively and qualitatively representative for the whole epiphytic populations.

These results, followed by later investigations on different plants must be useful as a technical approach for scientists and for water administrators in the river monitoring.

1. Laboratoire d'Ecologie des Eaux Continentales Méditerranéennes, case 331 IMEP-CNRS (URA 6116), Faculté des Sciences de Saint-Jérôme, Avenue Escadrille Normandie-Niemen, 13397 Marseille cedex 20, France.

E-mail: katia_comte@yahoo.fr

2. Laboratoire de Biomathématiques, case 462 IMEP-CNRS (URA 6116), Faculté des Sciences de Saint-Jérôme, Avenue Escadrille NormandieNiemen, 13397 Marseille cedex 20, France. 


\section{Introduction}

Depuis quelques années, la recherche du diagnostic de l'état de santé d'une rivière, entreprise par les limnologues et par les gestionnaires de l'eau, s'est développée en particulier, dans le but d'obtenir un matériel bio-indicateur spécifique des changements environnementaux.

Si l'utilité et l'efficacité de l'outil épilithique algal ne sont plus à démontrer aujourd'hui, le problème se pose lorsque celui-ci vient à manquer notamment dans des rivières qui se caractérisent par des fonds sablonneux, fortement peuplés de macrophytes.

Pendant un grand nombre d'années, l'étude de l'épiphytisme algal en rivière a été délaissée, en raison notamment d'un échantillonnage difficile et trop souvent aléatoire, accru par une grande variabilité saisonnière et une hétérogénéité des herbiers macrophytiques. Les quelques études menées en eau courante révèlent le fort degré de variation inhérent à la plante même, et semblent, en outre, indiquer une répartition aléatoire de la colonisation algale (Cattaneo \& Kalff 1979). Ces premières recherches sont souvent exprimées en terme de biomasse algale (Aloi 1990) ou en diversité spécifique totale après destruction de la plante-hôte par traitement chimique (Gough \& Woelkerling 1976, O'Quinn \& Sullivan 1983, Cazaubon 1989). Quelques autres, en revanche, nous renseignent sur la diversité de la communauté estimée "in situ" sur la plante-hôte, mais sans informer de l'état physiologique de la plante, de la localisation précise de l'organe foliaire prélevé, ni de la microrépartition de l'épiphyton sur le foliole (Allen 1971). C'est sans compter avec la complexité architecturale de la plante et les relations épiphytes/plante-hôte, lors de l'accrochage algal. C'est fort de ce constat, qu'a pu être entreprise la présente étude, qui concerne la microdistribution algale, sur le substrat vivant à différentes échelles d'observation, relançant ainsi la polémique fondée sur d'hypothétiques interrelations entre le complexe épiphytes/plante-hôte développées par bon nombre d'auteurs (Allen 1971, Allanson 1973, Blindow 1987, Burkholder \& Wetzel 1989) ou au contraire l'absence d'échanges cellulaires (Gough \& Gough 1981, Cattaneo 1978, Morin 1986, Cattaneo \& Kalff 1978).

Une première série de découpages a été réalisẹee à l'échelle de la plante, afin de prendre en compte le facteur individu, ainsi que la position et la face étudiée du foliole sur la feuille entière. Une deuxième série d'observations plus fines a été effectuée, à l'échelle du foliole, par un découpage longitudinal systématique réalisé sur toute sa longueur, ainsi que par une microzo- nation déterminée par la constitution même du foliole, avec la distinction du bord, du centre, de la nervure principale et de l'apex. Cette approche méthodologique apporte des informations précises quant à la distribution spatiale des communautés épiphytiques sur un support végétal très commun en milieux calcaires.

Un tel échantillonnage pourrait s'étendre à d'autres macrophytes aquatiques de divers systèmes lotiques en vue d'une meilleure connaissance de ce peuplement algal.

\section{Matériel et méthodes}

\subsection{Site d'étude}

L'étude a été réalisée sur une rivière méditerranéenne naturelle du sud-est de la France, la Sorgue, qui s'écoule au Nord-Est d'Avignon (Vaucluse) (Fig.1).

Elle prend sa source à Fontaine de Vaucluse, qui possède toutes les caractéristiques d'une source karstique vauclusienne (Puig 1987) dont un bassin versant très étendu et une isothermie annuelle avec des températures qui varient entre 11 et $14^{\circ} \mathrm{C}$.

La station se situe dans le secteur initial de la rivière, à environ $4,5 \mathrm{~km}$ de la source, et à quelques mètres en aval d'un petit affluent 'la Folie' qui amène un apport organique supplémentaire. Malgré ces rejets, la Sorgue demeure dans ce secteur une rivière de bonne qualité d'eau (classée 1A), selon la classification physico-chimique de Nisbet \& Verneaux 1970), très oxygénée et fraîche, à courant modéré à fort $\left(0,8-1 \mathrm{~m} \cdot \mathrm{s}^{-1}\right)$. Ces conditions favorisent le développement de grands herbiers d'Apium nodiflorum qui présentent un fort potentiel de recouvrement ( $70 \%$ de la surface du cours d'eau).

\subsection{Prélèvement macrophytique}

A. nodiflorum est l'espèce dominante du peuplement macrophytique de la Sorgue. Cette Apiacée présente des surfaces foliaires planes et étendues, ce qui facilite toute technique d'échantillonnage. La majorité des pieds d'Apium, située sur le bord des herbiers, provient du pied mère central. Chaque pied se compose de 4 à 6 feuilles, elles-mêmes constituées de plusieurs folioles (Fig. 2). Ces pieds sont supposés physiologiquement 'adulte' par le nombre $(\mathrm{n}=13)$ et la taille moyenne (longueur de $6 \mathrm{~cm}$ ) des folioles présents, ainsi que par une hauteur comprise entre 60 et $70 \mathrm{~cm}$. Quatre échantillons d'Apium, issus de 4 individus différents, ont été prélevés en tenant compte de l'homogénéité dés principaux paramètres physiques du milieu aquatique mesurés au niveau des plants, telle qu'une profondeur similaire $(75$ à $85 \mathrm{~cm})$, un ensoleillement 


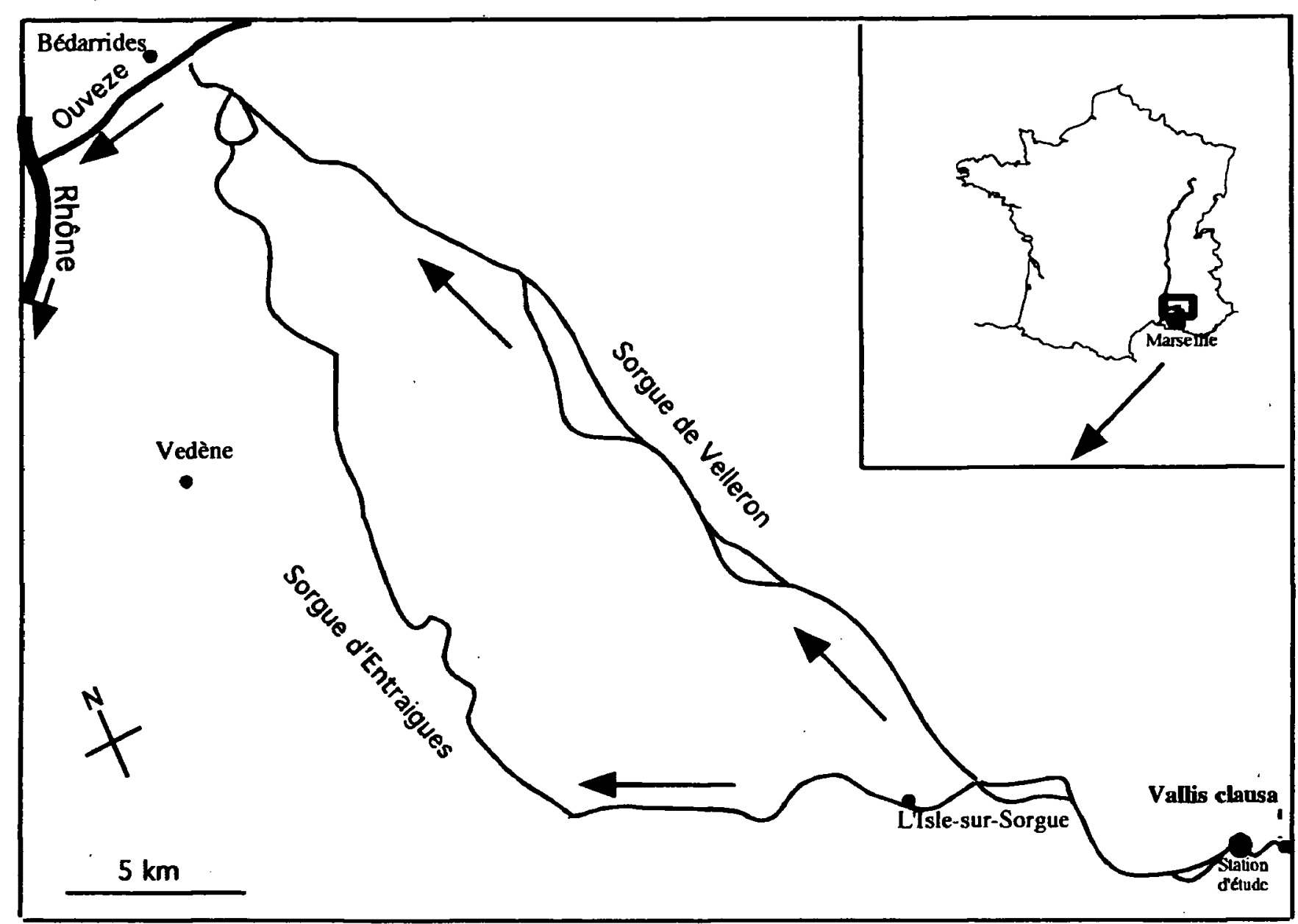

Fig. 1.Le site d'étude.

Fig. 1. The study area.

maximal, et une position comparable au sein de chaque herbier (sittation au bord et non au centre afin de réduire l'influence des frottements foliaires abrasifs, et d'un déficit de lumière enregistré au centre des herbiers massifs dans la station). Chaque pied est aussitôt fixé au formol neutre $(5 \%)$ et placé séparément dans un bocal en verre.

Deux campagnes de prélèvement ont été effectuées, pendant les 2 säisöìs: de forte croissance végétale, en automne (octobre 1997) et en été (juin 1998).

\subsection{Echantillonnage proposé}

Une feuille de chaque pied est prélevée et notée avec le numéro du pied (PL1 à PL4) dont elle est issue (Fig. 2). Deux séries de découpages sont alors réalisées au sein de ces 4 feuilles.

La première série, correspondant aux macrodécoupages, prend en compte la face du foliole - facl et fac2 - ainsi que sa position sur la hauteur totale de la feuille - Zon1, 2, $3-$ (Fig. 2). Ce premier découpa- ge permet l'obtention de 12 folioles par feuille, dont 6 sont étudiés par leur face supérieure et 6 par leur face inférieure.

La deuxième série, qualifiée de microdécoupages, permet la séparation du foliole en transects longitudinaux - Tr $0,1,2,3,4,5$ - et en microhabitats constitutifs de l'architecture foliaire $-\mathrm{B}, \mathrm{C}, \mathrm{N}-$. L'association de ces 2 découpages sur chaque foliole permet l'obtention de 16 microzones ( 3 microhabitats par transect, plus l'apex T0) par foliole (12 folioles/feuille) pour chacune des 4 feuilles et donc à l'observation totale de 768 microzones par série de prélèvement.

\subsection{Algues épiphytiques}

La présente étude est menée sur le 'vrai' épiphyton, c'est-à-dire sur les communautés algales fixées solidement au substrat (Snoejs 1994). Dans cette optique, chaque feuille est entreposée dans un récipient d'eau distillée et doucement rincée, afin d'extraire les algues 


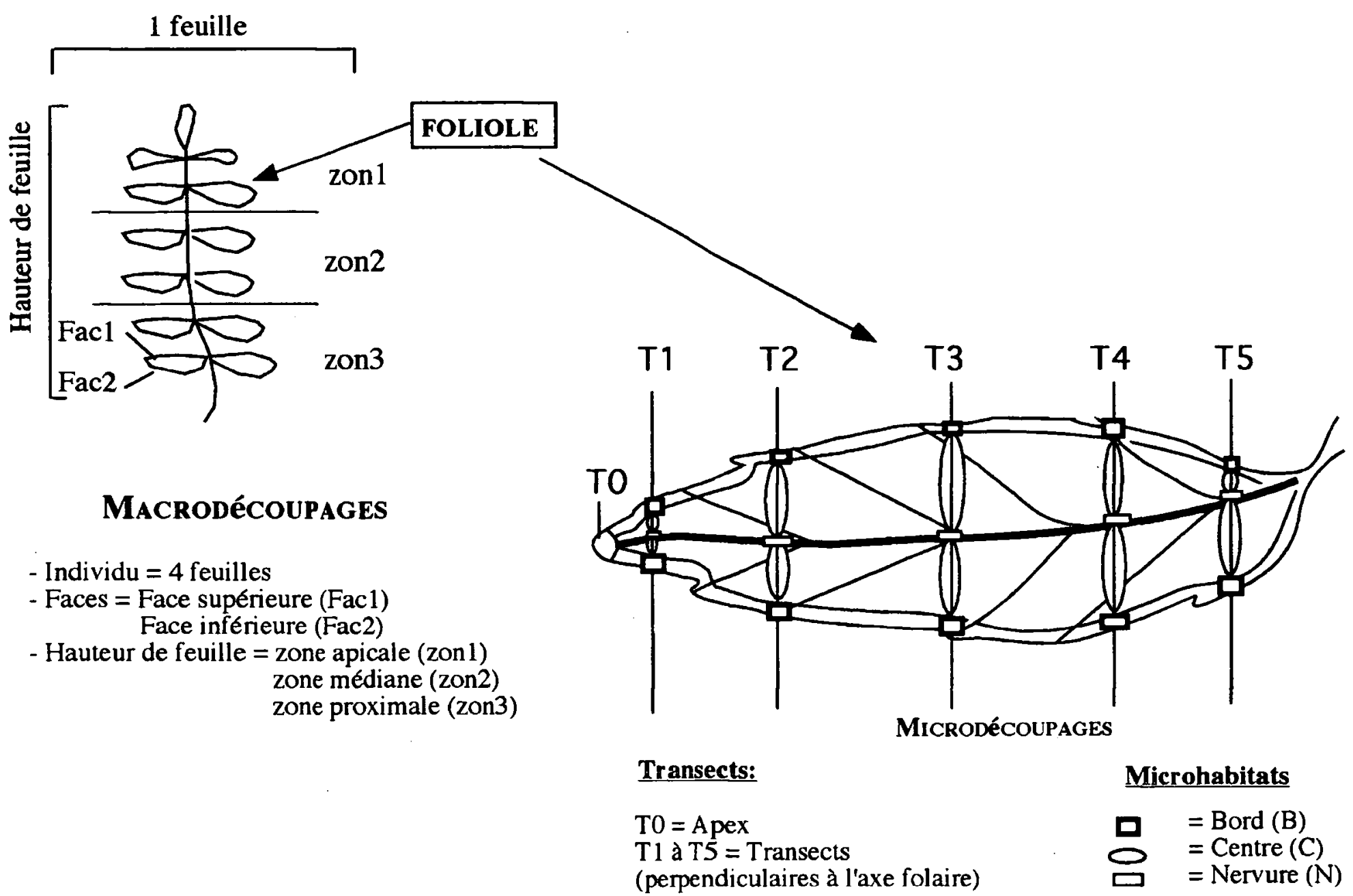

Fig. 2. Découpages réalisés sur un plant d'Apium nodiflorum.

Fig. 2. Cuttings carried out on Apium nodiflorum leaflets.

déposées passivement à la surface du macrophyte, appartenant au pseudoépiphyton (Goldsborough et al. 1986). Différentes techniques mécaniques ont été testées dans le but de réduire au maximum la perte d'épiphytes stricts. Seul un rinçage doux a permis d'obtenir un peuplement algal qualitativement proche de celui relevé lors des prélèvements 'in situ' (Comte 2001).

Les algues épiphytiques sont dénombrées au microscope droit 'Olympus' et identifiées 'in situ' avec les Süßwasserflora de Lange-Bertalot (Krammer \& Lange-Bertalot 1986, 1988, 1991a, b) pour les Diatomées et la flore des algues d'eau douces de Bourrelly (1966, 1968, 1970) pour les autres taxa.

En raison de l'épaisseur du support végétal qui rend délicate la détermination spécifique, cette observation est renforcée par un grattage successif des microzones de chaque foliole afin de recueillir les algues et de les identifier entre lame et lamelle (Comte 2001). Les diatomées sont préalablement grillées afin de faciliter la détermination. Le peuplement épiphytique spécifique de chaque microzone résulte de l'observation de 4 champs de microscope (réplicats) au grossissement $\mathrm{X} 100$, pris au hasard à l'intérieur de chaque microzone. La densité des communautés algales est exprimée en nombre de cellules. $\mathrm{mm}^{-2}$ de surface.

\subsection{Analyses statistiques}

Une Analyse en Composante Principale (A.C.P.) normée a été effectuée à chaque date, sur les effectifs bruts (nombre de cellules. $\mathrm{mm}^{-2}$ ) des espèces présentes dénombrées sur la totalité des microzones (768 relevés). Afin de souligner l'influence des découpages dans la répartition épiphytique, un tableau de modalités regroupant la totalité des microzones en variables de localisation (18 modalités au total avec les 6 transects, les 3 microhabitats, les 3 zones, les 2 faces et les 4 feuilles) a été projeté en éléments supplémentaires sur les axes factoriels (Figs 3, 4). Les analyses multivariées ont été réalisées sur le logiciel Statoscope 1.7 version 1999. Les taxa sont inventoriés et codés pour 

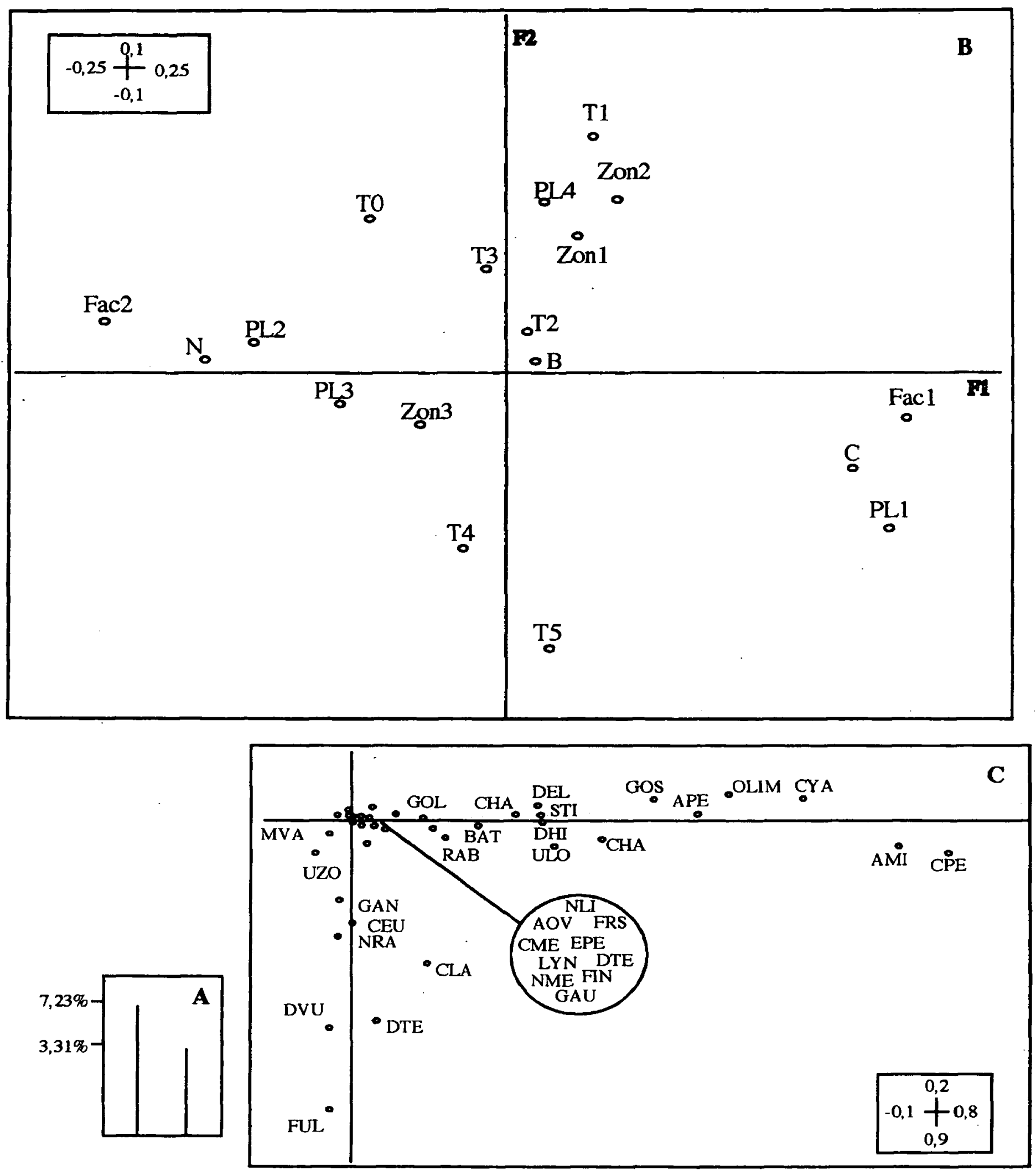

Fig.3. Analyse en Composante Principale (A.C.P.) sur les données d'octobre 1997. A) Carte des valeurs propres. B) Carte factorielle (F1XF2) des variables de localisation (ou modalités) représentatives des découpages multiples sur $A$. nodiflorum.

Fig. 3. Principal Component Analysis (P.C.A.) on October 1997 data. A) Eigenvalues. B) Factorial map (F1 X F2) of localisation variables representative of the multiple cuttings on A. modiflorum. C) Factorial map of epiphytic species present on all the microzones. 

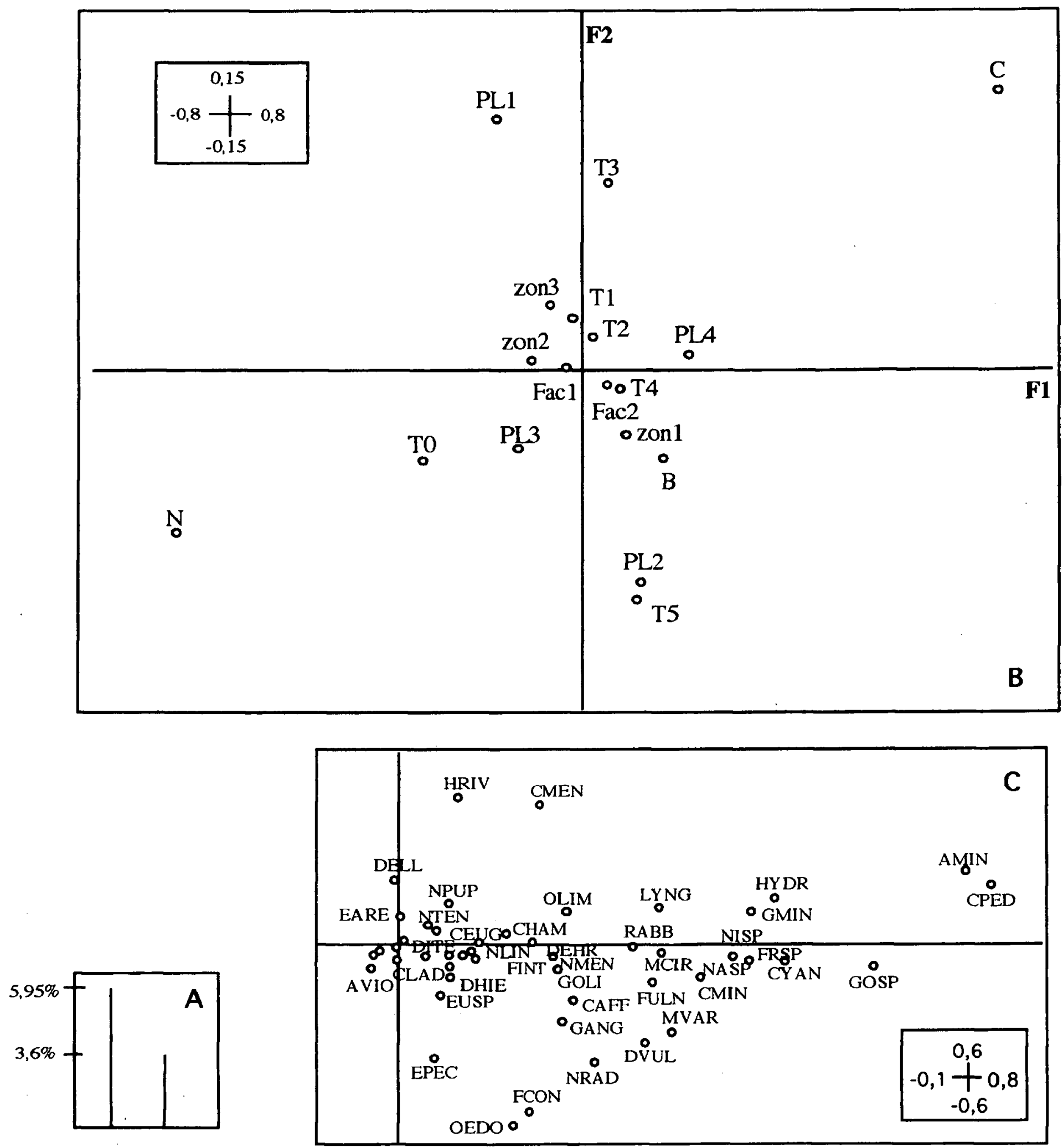

Fig.4. Analyse en Composante Principale (A.C.P.) de juin 1998. A) Carte des valeurs propres. B) Carte factorielle (F1XF2) des variables de localisation, représentatives des découpages multiples sur $A$. nodiflorum. C) Carte factorielle F1XF2) des espèces épiphytiques présentes sur la totalité des microzones.

Fig. 4. Principal Component Analysis (P.C.A.) on june 1998 data. A) Eigenvalues. B) Factorial map (F1 X F2) of localisation variables representative of the multiple cuttings on A. modiflorum. C) Factorial map of epiphytic species present on all microzones. 
l'analyse (Tableau 1). Les modalités sont notées dans les figures 3 et 4 .

L'étude de la répartition spatiale du peuplement sur les différents transects a été analysée au moyen des tests non paramétriques à comparaisons multiples (variables dépendantes $>3$ ) de Friedman (1954) et de Wilcoxon ( 2 variables dépendantes - Conover 1971), grâce au logiciel StatView 4.5 (Roth et al. 1995).

Un test d'ordination des tendances croissantes et décroissantes de Jonckeere-Terpstra (Siegel \& Castellan 1988) a été appliqué sur ces données pour détecter un gradient du peuplement en fonction de l'éloignement progressif des 5 transects de l'apex.

\section{Résultats}

\subsection{Constitution du peuplement algal sur 2 saisons}

Le peuplement épiphytique total se compose de 64 espèces - 40 espèces en automne et 58 en été - (Tableau 1). Quatre groupes d'algues sont représentés avec, en octobre, une dominance de Diatomophycées $(50,9 \%)$, liée à la fraicheur des eaux et à la vitesse du courant. Les Cyanobactéries représentent $47,7 \%$ du peuplement, les Chlorophycées $1,3 \%$, et les Rhodophycées $0,2 \%$. En juin, les Cyanobactéries prolifèrent avec une abondance relative de $69,9 \%$, les Diatomophycées ne représentent plus que $28,8 \%$, les Chlorophycées $<0,1 \%$, et les Rhodophycées $1,3 \%$. La densité totale varie de manière importante avec un dénombrement cellulaire 2 fois plus élevé en moyenne en octobre qu'en juin, en raison notamment de la forte croissance macrophytique et du développement des algues en dérive en juin 1998.

\subsection{Macrorépartition des communautés algales à l'échelle de la feuille}

En octobre, la répartition spatiale des espèces épiphytiques en fonction des variables de localisation est représentée par l'A.C.P. de la figure 3. Les 2 premiers axes de l'analyse sont représentés par des valeurs propres supérieures à 2,34 pour l'axe $1(7,23 \%$ d'inertie), et de 1,76 pour l'axe 2 (3,31\% d'inertie).

La carte factorielle (Fig. 3b) met en évidence une opposition de la feuille Pl1 (corrélée positivement à l'axe 1) avec les autres feuilles (corrélées négativement sur cet axe). Cette hétérogéneité individuelle étant confirmée par les tests de Friedman (avec $p<0,001$ ) : Tableau 3). S'ajoute à cette variabilité, une homogénéité quantitative du peuplement épiphytique sur les 3 zones de la feuille, traduit par une faible corrélation aux axes (Fig.3) et un test de Friedman non significatif ( $p>0,05$ : Tableau 3).
En revanche, les faces sont disposées le long de l'axe 1 , avec la face supérieure positivement corrélée à l'axe 1 et opposée à la face inférieure. La face supérieure étant en effet significativement plus colonisée que la face inférieure $(\mathrm{p}<0,001)$.

La carte factorielle des espèces (Fig. $3 c$ ) révèle non seulement la dominance très marquée de quelques espèces dans le peuplement (Cocconeis pediculus CPED, Achnanthes minutissima AMIN, Chlorogloea sp. CYAN et Gomphonema sp. : GOSP fortement corrélées à l'axe 1), mais également une majorité d'algues communes, à faibles effectifs, située près du centre d'inertie (Fig. 3).

Si les variations de densité algale sont réelles au sein de chaque individu macrophytique, il n'en est pas de même quant à la structure de la communauté épiphytique (Tableau 3). En effet, les tests de Friedman ne révèlent pas de différences qualitatives significatives du peuplement algal sur les 4 feuilles $(p>0,05)$. La richesse spécifique reste homogène quelle que soit la zone du foliole étudiée (Tableau 3). En revanche, la face supérieure possède un peuplement plus riche et plus varié que la face inférieure.

L'A.C.P. de la figure 4 illustre la disposition des variables de localisation des espèces récoltées en juin 1998. Les premiers axes sont représentés par des valeurs propres de 3,94 pour l'axe 1 (soit $5,97 \%$ d'inertie), et de 1,88 pour l'axe $2(3,6 \%$ d'inertie).

Des variations quantitatives et qualitatives semblables au niveau des 4 feuilles sont également relevées en juin 1998 (Fig. 4 a) et confirmées par les tests non paramétriques (Tableau 2). En revanche, aucune différence de richesse spécifique et de densité n'est retrouvée sur les 12 folioles de la même feuille, et ce, quelque soit la zone et de la face étudiée (Tableau 2).

La carte représentant la projection des espèces (b), met en évidence la dominance des 4 mêmes espèces d'algues citées précédemment, avec cependant une dispersion du nuage d'espèces le long de l'axe 1 et donc une répartition densitaire plus équilibrée au sein des autres communautés algales.

\subsection{Microrépartition algale à l'échelle du foliole}

Les découpages en transects et en microhabitats sont fortement corrélés aux axes 1 et 2 de l'A.C.P. pour le peuplement automnal. Les 3 microhabitats (Hab 1 à 3 ), respectivement, le bord, le centre et la nervure centrale, sont répartis le long de l'axe 1. La nervure est corrélée négativement à l'axe et opposée au centre (corrélé positivement) (Fig. 3a). Les tests de Friedman effectués sur la densité algale totale dénombrée, révèlent de 
Tableau 1. Liste des algues épiphytiques (en présence/absence) relevées sur la totalité des folioles d'Apium nodiflorum. Les codes en gras correspondent aux 4 espèces dominantes.

Table 1. Epiphytic populations (presence/absence) noted on the whole leaflets of Apium nodiflorum. The codes with bold type correspond to the 4 dominant species.

\begin{tabular}{|c|c|c|}
\hline $\begin{array}{l}\text { Epiphyton } \\
\text { BACILLARIOPHYCÉES } \\
\text { Achnanthes minutissima Kütz. AMIN } \\
\text { Achnanthes lanceolata (Breb.) Grun. ALAN } \\
\text { Amphora pediculus (Kütz.) Grun. APED } \\
\text { Amphora ovalis Kütz. AOVA } \\
\text { Cocconeis pediculus Ehr. CPED } \\
\text { Cocconeis placentula var. euglypta (Ehr.) Grun. CEUG } \\
\text { Cyclotella meneghiniana Kütz. CMEN } \\
\text { Cymbella affinis Kütz. CAFF } \\
\text { Cymbella cistula (Ehr.) Kirchner CCIS } \\
\text { Cymbella minuta Hile Rab. CMIN } \\
\text { Denticula tenuis Kütz. DTEN } \\
\text { Diatoma ehrenbergii Kütz. DEHR } \\
\text { Diatoma hiemale (Roth.) Heiberg DHIE } \\
\text { Diatoma tenuis Kütz DITE } \\
\text { Diatoma vulgaris Bory DVUL } \\
\text { Diploneis elliptica Kütz. DELL } \\
\text { Eunotia pectinalis (Kütz.)Rab. EPEC } \\
\text { Eunotia sp EUSP } \\
\text { Ellerbeckia arenaria (Moore) Crawford EARE } \\
\text { Fragilaria construens (Ehr.) Grun. FCON } \\
\text { Fragilaria crotonensis Kitton FCRO } \\
\text { Fragilaria intermedia Desmazières FINT } \\
\text { Fragilaria ulna (Nitzsch.) Lang.Bertalot FULN } \\
\text { Fragilaria sp. FRSP } \\
\text { Gomphonema acuminatum Ehr. GACU } \\
\text { Gomphonema augur Ehr. GAUG } \\
\text { Gomphonema angustatum (Kütz.) Rab. GANG } \\
\text { Gomphonema angustum Ag. GTUM } \\
\text { Gomphonema minutum (Ag.) Ag. GMIN } \\
\text { Gomphonema olivaceum (Horn.) Breb. GOLI } \\
\text { Gomphonema sp. GOSP } \\
\text { Gyrosigma acuminatum (Kütz.) Rab. GYAC } \\
\text { Melosira varians Ag. MVAR } \\
\text { Meridion circulare (Grev.) Ag. MCIR } \\
\text { Navicula cryptotenella Lange-Bertalot NTEN } \\
\text { Navicula lanceolata (Ag.) Ehr. NLAN } \\
\text { Navicula menisculus (Schumann) Grun. NMEN } \\
\text { Navicula pupula Kütz. NPUP } \\
\text { Navicula radiosa Kütz. NRAD } \\
\text { Navicula tripunctata (Muller) Bory NTRI } \\
\text { Navicula sp. NASP } \\
\text { Nitzschia linearis (Ag.) W. Smith NILI } \\
\text { Nitzschia palea (Kütz.) W. Smith NPAL } \\
\text { Nitzschia sp. NISP } \\
\text { Rhoicosphenia abbreviata (Ag.) Lange-Bertalot RABB } \\
\text { Stauroneis sp. STSP } \\
\text { CHLOROPHYCEES } \\
\text { Stigeoclonium sp. Kütz. STIG } \\
\text { Chaetophora sp. CHAE }\end{array}$ & $\begin{array}{l}+ \\
+ \\
+ \\
+ \\
+ \\
+\end{array}$ & $\begin{array}{l}+ \\
+ \\
+ \\
+ \\
+ \\
+ \\
+ \\
+ \\
+ \\
+ \\
+ \\
+ \\
+ \\
+ \\
+ \\
+ \\
+ \\
+ \\
+ \\
+ \\
+ \\
+ \\
-- \\
\because \\
+ \\
+ \\
+\end{array}$ \\
\hline
\end{tabular}


Tableau 1. Suite.

Table 1. Continued.

\begin{tabular}{|l|c|c|}
\hline Cladophora glomerata ( L.) Kütz. CLAD & + & + \\
Closterium sp. CLOS & + & + \\
Oedogonium sp. OEDO & + & + \\
Pearsoniella variabilis Fritsch. Rich. PVAR & + & + \\
Ulothrix zonata UZON & + & + \\
Ulothrix sp. ULO1 & & + \\
CYANOPHYCEES & & + \\
Anabaena sp. ANAB & + & + \\
Chamaesiphon incrustans CHAM & + & + \\
Chlorogloea spl CYAN & + & + \\
Chlorogloea sp2 CHLO & & + \\
Hydrococcus sp. HYDR & + & + \\
Lyngbya sp. LYNG & + & + \\
Oscillatoria limosa Ag. OLIM & & + \\
RHODOPHYCEES & & + \\
Audouinella violacea (Kütz.) Hamel AVIO & + & + \\
Hildenbrandia rivularis (Roth.) Ag. HRIV & + & + \\
Bangia atropurpurea (Liebm.) Ag. BATR & & \\
\hline
\end{tabular}

fortes différences densitaires entre la nervure peu colonisée (environ 3000 cellules. $\mathrm{mm}^{-2}$ ), et le centre surpeuplé (effectifs supérieurs à $9000 . \mathrm{mm}^{-2}$ ). L'apex représente une microzone particulière, très peu colonisée par les algues (moins de 700 cellules. $\mathrm{mm}^{-2}$ ). La même répartition algale s'observe en juin, avec des différences marquées entre la nervure et le reste du foliole (Tableaux 2, 3).

A cette variabilité densitaire s'ajoutent des différences qualitatives significatives, avec une diversité algale maximale sur le bord et le centre du foliole (Tableau 3). L'apex et la nervure sont peuplés par quelques espèces pionnières et dominantes telles qu'Achnanthes minutissima et Cocconeis pediculus, également communes aux deux saisons.

Les transects T0 à T5 sont plus fortement corrélés à l'axe 2, avec une opposition entre les transects apicaux $\mathrm{T} 0$ et $\mathrm{T} 1$ (corrélés positivement) et les transects proximaux T4 et T5 (corrélés négativement). Ces résultats, renforcés par le test de Jonckeere-Terpstra ( $p<0,001$ sur un test de $n=400$ permutations) mettent en évidence un gradient longitudinal décroissant de la densité algale totale sur chaque foliole et en revanche, une richesse spécifique inversément proportionnelle à la densité cellulaire (avec une probabilité de tendance croissante: $p<0,001$ ). Ces deux tendances sont identiques aux deux saisons.

\section{Discussion}

L'étude de la répartition épiphytique sur Apium nodiflorum, montre qu'au cours de 2 périodes de forte croissance végétale, le peuplement algal évolue de façon dynamique dans la rivière et tend à confirmer l'observation d'Allen (1971), Allanson (1973) et Wetzel (1983) selon laquelle la nature lisse du substrat macrophytique entraîne une succession algale complexe au cours d'un cycle annuel.

Ce modèle n'est en rien figé, bien qu'une forte base d'espèces algales ( $A$. minutissima et $C$. pediculus) appartenant au vrai épiphyton, demeure constante et dominante sur la plante au cours des saisons (Pozzobon \& Telle 1995, Tesolin \& Tell 1996, Acs 1991, Watanabe et al. 1988). Seul le cortège floristique accompagnant ces espèces varie dans le temps, avec notamment de fortes croissances de cyanobactéries en juin 1998.

La forte variabilité enregistrée à l'échelle de l'individu macrophytique montre à quel point il est difficile d'estimer l'état physiologique (Campbell \& Clarck 1983, Hargeby 1990) et le 'passé' de chaque plant dans leurs herbiers respectifs. Les paramètres biotiques ajoutés aux conditions environnementales entrainent inéluctablement une colonisation et un développement algal différent selon l'individu étudié, en dépit d'une similitude de mesures morphométriques. 
Tableau 2. Moyenne et écart-type des densités totales (nombre de cellules $/ \mathrm{mm}^{2}$ ) relevées aux 2 dates et analysées par les tests de Friedman et de Wilcoxon sur les faces foliaires. $\left({ }^{* * *} \mathrm{p}<0.001 ;{ }^{* *} \mathrm{p}<0.01 ;{ }^{*} \mathrm{p}<0.05=\right.$ différences significatives ; NS $\mathrm{p}>0.05=$ non significatives).

Table 2. Mean and standard deviation of the total density (number of cells $/ \mathrm{mm}^{2}$ ) recorded on the two dates over the different transects and analysed by Friedman's and Wilcoxon's tests (on the leaf sides). $\left({ }^{* * *} \mathrm{p}<0.001 ;{ }^{* *} \mathrm{p}<0.01 ;{ }^{*} \mathrm{p}<0.05=\right.$ significant differences; NS $\mathrm{p}>0.05=$ non significant).

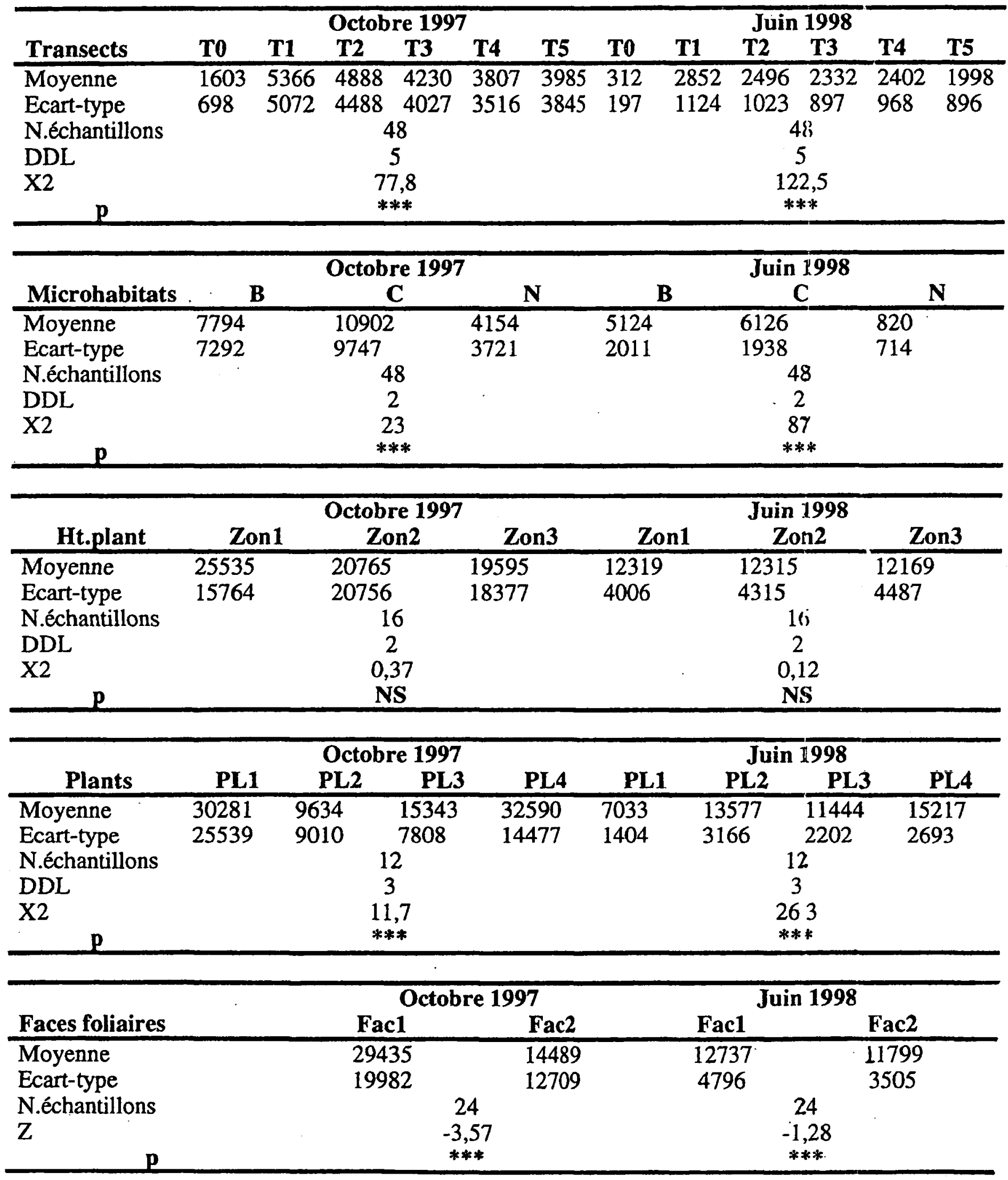


Tableau 3. Moyenne et écart-type de la richesse spécifique algale relevées aux 2 dates et analysées par les tests de Friedman et de Wilcoxon sur les faces foliaires. $\left({ }^{* * *} \mathrm{p}<0.001 ;{ }^{* *} \mathrm{p}<0.01 ;^{*} \mathrm{p}<0.05=\right.$ différences significatives ; NS $\mathrm{p}>0.05=$ non significatives).

Table 2. Mean and standard deviation of the algal specific richness recorded on the two dates over the different transects and analysed by Friedman's and Wilcoxon's tests (on the leaf sides). $\left({ }^{* * *} p<0.001 ;{ }^{* *} p<0.01 ;{ }^{*} p<0.05=\right.$ significant differences ; NS p $>0.05=$ non significant $)$.

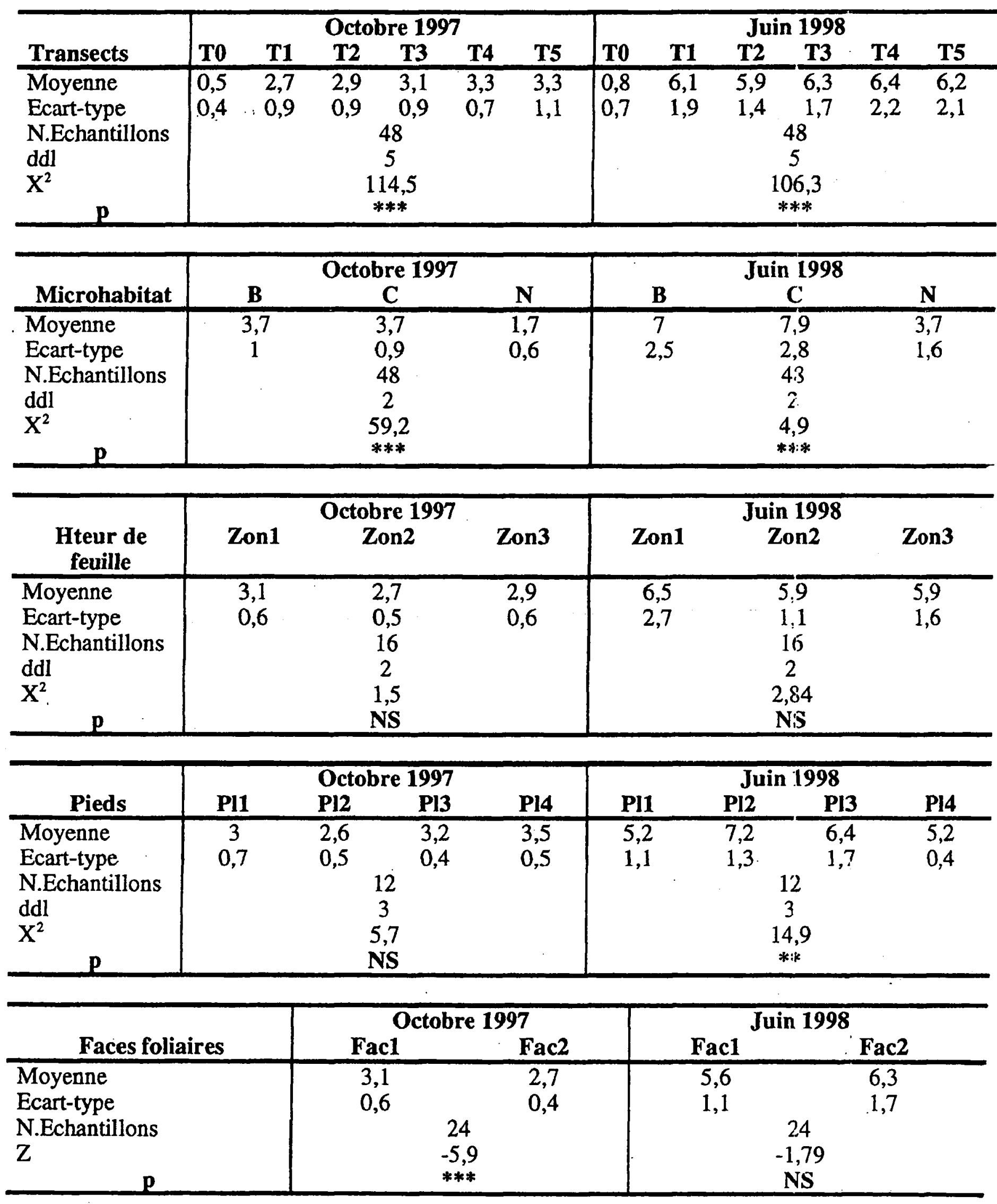


Outre cette forte variabilité individuelle, des modèles de structures épiphytiques se dessinent sur les différents folioles constitutifs d'une feuille. Cette homogéneité algale, relevée sur la hauteur totale des feuilles, peut être attribuée à la transparence des eaux de la Sorgue, qui favorise la pénétration des rayons lumineux sur toute la colonne d'eau. Néammoins si une quantité de lumière suffisante pour permettre le développement algal atteint les zones les plus profondes, on ne peut exćlure que la qualité lumineuse, exprimée en longueurs d'onde pourrait jouer un rôle dans la répartition spatiale et expliquer ainsi, les différences significatives de densité et de diversité algales sur les faces foliaires entre l'automne et l'été.

Le nombre élevé de folioles analysés révèle une disposition non aléatoire des algues, liée à l'architecture de la feuille elle-même, avec la nervure et l'apex moins hospitaliers que le limbe. La distinction de $3 \mathrm{mi}-$ crohabitats (bord, centre, nervure) influe directement sur l'implantation des espèces pionnières de façon plus discriminante que la différenciation individuelle d'un plant. Il est vraissemblable que le relief de la nervure expose davantage la communauté à l'abrasion particulaire due au courant et aux matières en suspensions transportées, ainsi qu'à la pression de broutage de macro-invertébrés et de poissons. Il n'en demeure pas moins que l'absence quasi totale d'épiphytes sur cette partie de foliole pourrait aussi résulter d'une absence d'échanges gazeux au niveau des cellules de la nervure. On ne peut également écarter l'influence des cellules indifférenciées de l'apex sur l'installation algale, même si la croissance foliaire est supérieure au temps de colonisation épiphytique, expliquant ainsi la faible variété et densité algales sur cette zone.

Une différenciation marquée apparaît sur l'axe longitudinal du foliole (exprimé par les 5 transects) quels que soient la zone de la feuille et l'individu étudiés. A partir de l'apex, s'établit un gradient de colonisation caractérisé par une réduction progressive de la densité cellulaire des espèces dominantes pionnières au profit d'une organisation algale plus évoluée, représentée par des espèces compétitrices diversifiées mais peu fréquentes ( $<1 \%$ du peuplement total). Des résultats similaires, liés à la maturité foliaire, ont été décrits par Cattaneo (1978) à une échelle d'observation différente, sur les feuilles entières de plantes. S'il semble envisageable, de par la croissance végétale des folioles, que la zone apicale est de constitution plus récente que les deux autres, on peut néammoins supposer que les transects T5 représentent un espace particulier plus rigide, en raison notamment de la jonction avec le pétiolule, qui les exposent d'autant moins aux forces du courant. De nombreux travaux (Peterson et al. 1990, Cazaubon \& Loudiki 1986, Fayolle et al. 1998, Acs 1900) ont mis en évidence l'impact principal de l'hydrodynamisme sur les réponses spécifiques des algues dans les rivières. Cette influence serait prépondérante à celle de la maturité foliaire, ce qui pourrait expliquer la variabilité des populations épiphytiques observée à l'échelle du foliole et non pas à l'échelle de la feuille entière. Ainsi, le fort courant $\left(>80 \mathrm{~cm} . \mathrm{s}^{-1}\right)$ inhiberait l'installation de la plupart des épiphytes sur toutes les parties apicales des folioles, les plus exposées aux mouvements hydriques et ce, quels que soient leur âge et leur disposition foliaire.

Ces premiers résultats, confirmés aux 2 saisons de prélèvement, révèlent l'existence d'une répartition algale dynamique et non stochastique, dépendante de la surface et de l'échelle d'observation étudiée. Au vu des premières observations à l'échelle de la plante, il semble moins nécéssaire de multiplier le nombre de folioles sur une même feuille (absence de différences qualitatives et quantitatives), que d'étudier plusieurs feuilles issues de pieds différents (variabilité individuelle très significative). De même, il semble que la face supérieure possède une population épiphytique plus complexe et plus variée que la face inférieure selon la saison analysée.

Ainsi, au même titre que la méthode de l'étude de l'épilithon en rivière, qui suggère la prise en compte minimale de 5 cailloux différents pour l'obtention d'un échantillon représentatif (Cazaubon 1998, CEMAGREF 1982, Kelly et al.1998), l'analyse épiphytique pourrait être menée sur un minimum de 4 plants différents afin de reduire une double variabilité, liée aux conditions environnementales et surtout à l'état physiologique complexe du support vivant.

\section{Remerciements}

Les auteurs remercient tout particulièrement les Drs. Evelyne Franquet et Nadira Taïeb pour les nombreuses critiques apportées au manuscrit, et expriment toute leur gratitude à la Fédération de Pêche du Vaucluse pour avoir financé une partie de l'étude.

\section{Références}

Acs E. 1991. - Investigation of periphytic algae in the Danube at Göd (1669 river km, Hungary). Arch. Hydrobiol. Suppl. Bd. 89., Algol. Studies, 62 : 47-67.

Acs E. 2000. - Short-term colonization sequence of periphyton on glass slides in a large river (River Danube, near Budapest). Archiv. Hydrobiol., Algol. Studies, 100 : 135-156.

Allanson B.R. 1973. - The fine structure of the periphyton of Chara sp. and Potamogeton natans from Wytham pond, Oxford. Freshwat. Biol., 3 : 535-541.

Allen H. 1971. - Primary productivity, chemo-organotrophy and nutritional interactions of epiphytic algae and bacteria on macrophytes in the littoral of a lake. Ecol. Monogr., $41: 97-127$. 
Aloi J.E. 1990. - A critical review of recent freshwater periphyton fields methods. Can. J. Fish. Aquat. Sci., $47: 656-670$.

Blindow I. 1987. - The composition and density of epiphyton on several species of submerged macrophytes. The neutral substrate hypothesis tested. Aquat. Bot., 29 : 157-168.

Bourrelly P. 1966. - Les algues d'eau douce. Initiation à la systématique. 1 : Les algues vertes. Boubé, Paris : 511 p.

Bourrelly P. 1968. - Les algues d'eau douce. Initiation à la systématique. 2 :Les algues jaunes et brunes. Boubée, Paris : 438 p.

Bourrelly P. 1970. - Les algues d'eau douce. Initiation à la systématique. 3 : Les algues bleues et rouges, les Euglèniens, Péridiniens et Cryptomonadines. Boubée, Paris : $512 \mathrm{p}$.

Burkholder J.M. \& Wetzel R.G. 1989. - Microbial colonization on natural and artificial macrophytes in a phosphorus-limited, hardwater lake. J. Phycol., $25:$ 55-65.

Campbell J.M. \& W.J. Clarck. 1983. — Effect of microhabitat heterogeneity on the spatial dispersion of small plant-associated invertebrates. Freshwat. Invert. Biol., 2 : 180-185.

Cattaneo A. 1978. - The microdistribution of epiphytes on the leaves of natural and artificial macrophytes. Br. Phycol. J., 13 : 183-188

Cattaneo A. \& Kalff J. 1978. - Seasonal changes in the epiphyte community of natural and artificial macrophytes in Lake Memphrenagog. Hydrobiologia, $60: 135-144$.

Cattaneo A. \& Kalff J. 1979. - Primary production of algae growing on natural and artificial aquatic plants : A study of interactions between epiphytes and their substrate. Limnol. Oceanog., 24 (6) : 1031-1037.

Cazaubon A. \& Loudiki M. 1986. - Microrépartition des algues épilithiques sur les cailloux d'un torrent corse, le Rizzanese. Ann. Limnol., 22 (1) : 3-16.

CEMAGREF. 1982. - Etude des méthodes biologiques quantitatives d'appréciation de la qualité des eaux. - Rapport Q.E. Lyon. A.F.B. Rhône-Méditerranée-Corse : 218 p.

Comte K. 2001. - Etude de la variabilité épiphytique en rivière méditerranéenne. Comparaison des communautés microphytiques sur différentes plantes-hôtes. Thèse de $3^{\text {ème }}$ cycle, Aix-Marseille III : $243 \mathrm{p}$.

Conover W.J. 1971. - Practical Nonparametric statistics. John Wiley \& Sons, New York : $462 \mathrm{p}$

Fayolle S., Cazaubon A., Comte K. \& Franquet E. 1998. - The Intermediate Disturbance Hypothesis : application of this concept to the response of epilithon in a regulated Mediterranean river (Lower-Durance, southeastern France). Arch. Hydrobiol., 143 : 57-77.

Friedman A.R. 1954. - A distribution-free k-sample test against ordered alternatives. Biometrika, $41: 133-145$.

Golsborough L.G., Robinson G.G.C. \& Gurney S.E. 1983. - An enclosure/substratum system for in situ ecological studies of periphyton. Arch. Hydrobiol., 106 (3) : 373-393.

Gough S.B. \& Gough L.P. 1981. - 'Comment on Primary production of algae growing on natural and artificial aquatic plants : a study of interactions between epiphytes and their substrates (Cattaneo and Kalff)'. Limnol. Oceanog., 26 : 987-988.

Gough S.B. \& Woelkerling W.J. 1976. - On the removal and quantification of algal aufwuchs from macrophytes hosts. Hydrobiologia, 48 : 203-207.

Hargeby A., 1990. - Macrophyte associated invertebrates and the effect of habitat permanence. Oikos, $57: 338-346$.
Kelly M.G, Cazaubon A., Coring E., Dell'Uomo L., Ector A., Goldsmith B., Guasch H., Hürlimann J., Jarlman A., Kaweka B., Kwandrans J., Laugaste R., Lindstrom E.A., Leitao M., Marvan P., Padisak J., Pipp E., Prygiel J., Rott E., Sabater S., Van Dam H., Vizinet J. 1998. - Recommendations for the routine sampling of diatoms for water quality assessments in Europe. J. Appl. Phycol., $10(2): 15-224$.

Krammer K. \& Lange-Bertalot H. 1986. - Bacillariophyceae 1 : Naviculaceae. - Süßwasserflora von Mitteleuropa, H. Ettl, J. Gerloff, H. Heynig \& D. Mollenhauer (eds.) ; Band 2/ : G. Fisher Verlag, Stuttgart : $876 \mathrm{p}$.

Krammer K. \& Lange-Bertalot H. 1988. - Bacillariophyceae 2 : Bacillariaceae, Epithemiaceae, Surirellaceae. - Süßwasserflora von Mitteleuropa, H. Ettl, J. Gerloff, H. Heynig \& D. Mollenhauer (eds.) ; Band $2 / 2$ : G. Fisher Verlag, Stuttgart : 596 p.

Krammer K. \& Lange-Bertalot H. 1991a. - Bacillariophyceae 3 : Centrales, Fragilariaceae, Eunotiaceae. - Sü $\beta$ wasserflora von Mitteleuropa, H. Ettl, J. Gerloff, H. Heynig \& D. Mollenhauer (eds.) ; Band 2/3 : G. Fisher Verlag, Stuttgart: 600 p.

Krammer K. \& Lange-Bertalot H. 1991b. - Bacillariophyceae 4 : Achnanthaceae. Kritische Ergäzungen zu Navicula (Lineolatae) und Gomphonema. - Sübwasserflora von Mitteleuropa, H. Ettl, J. Gerloff, H. Heynig \& D. Mollenhauer (eds.) ; Band 2/4 : G. Fisher Verlag, Stuttgart : $437 \mathrm{p}$.

Morin J.O. 1986. - Initial colonization of periphyton on natural and artificial apice of Myriophyllum heterophyllum. Freshwat. Biol., $16: 685-694$.

Nisbet M. \& Verneaux J. 1970. - Composantes chimiques des eaux courantes. Discussion et proposition de classes en tant que bases d'interprétation des analyses chimiques. Ann. Limnol., 6 : 161-190.

O'Quinn R. \& Sullivan M.J. 1983. - Community structure dynamics of epilithic and epiphytic diatoms in a Mississippi stream. $J$. Phycol, 19 : 119-128.

Pozzobon M.V. \& Telle G. 1995. - Estructura y dinàmica de la comunidad perifica sobre Ricciocarpos natans (Hepaticae) de la Laguna de los Padres (Buenos Aires, Argentina). Bol. Sec. Argent. Bot., $30: 199-208$.

Puig J.M. 1987. - Le système karstique de la Fontaine de Vaucluse. Thèse $3^{\text {ème }}$ cycle. Univ. d'Avignon : $207 \mathrm{p}$.

Roth J., Haycock K., Gagno J., Soper C. \& Caldarola J. 1995. L'intégré des analyses de données. Stat view 4.5. Abacus Concepts, Inc. California, USA : 75p.

Siegel S. \& Castellan Jr. N.J. 1988. - Non parametric statistics for the behavioral sciences. Mc Graw Hill, New York : 399 p.

Snoejs P. 1994. - Distribution of epiphytic diatom species composition, diversity and biomass on different macroalgal hosts along seasonal and salinity gradients in a Baltic sea. Diatom Res., 9 (1) : 189-211.

Tesolin G. \& Tell G. 1996. - The epiphytic algae on floating macrophytes of a Paranà river floodplain lake. Hydrobiologia, 333 : 111-120.

Watanabe T., Capblancq J. \& Dauta A. 1988. - Utilisation des bioessais «in situ» (substrats artificiels) pour caractériser la qualité des eaux de rivière à l'aide du périphyton. Ann. Limnol., 24 : 111-125.

Wetzel R.G. 1983. - Periphyton of freshwater ecosystems. Proceed. 1st. int. workshop on periphyton of freshwater ecosystems, The Hague. W. Junk Publishers : 339 p. 